\title{
AMPK activation serves a critical role in mitochondria quality control via modulating mitophagy in the heart under chronic hypoxia
}

\author{
HUAGANG ZHANG ${ }^{*}$, BO LIU*, TIANBO LI, YUN ZHU, GUIPING LUO, YUNHAN JIANG, \\ FUQIN TANG, ZHAO JIAN and YINGBIN XIAO ${ }^{1}$ \\ Department of Cardiovascular Surgery, Xinqiao Hospital, The Third Military Medical University, \\ Chongqing 400037, P.R. China
}

Received December 24, 2015; Accepted October 17, 2017

DOI: $10.3892 /$ ijmm.2017.3213

\begin{abstract}
Mitochondrial biogenesis is one of the generally accepted regulatory mechanisms in the heart under chronic hypoxia. The precise quantity and quality control of mitochondria is critical for the survival and function of cardiomyocytes. Mitochondrial autophagy, also known as mitophagy, which selectively eliminates dysfunctional and unwanted mitochondria, is the most important type of mitochondrial quality control. However, the detailed molecular mechanisms of mitophagy in cardiomyocytes have been largely undefined. The present study investigated the role of adenosine 5'-monophosphate-activated protein kinase (AMPK) in mitophagy regulation in cardiomyocytes under chronic hypoxia. H9c2 cells were cultured under hypoxic conditions $\left(1 \% \mathrm{O}_{2}\right)$ for different time periods. Mitochondrial biogenesis was confirmed and hypoxia was found to induce the collapse of mitochondrial membrane potential $(\Lambda \Psi \mathrm{m})$ and increase the number of dysfunctional mitochondria. As expected, mitochondrial autophagy was increased significantly in cardiomyocytes exposed to hypoxic conditions for $48 \mathrm{~h}$. AMPK was activated under hypoxia. Notably, when the activation of AMPK was enhanced by the AMPK agonist AICAR, mitochondrial autophagy was increased accordingly. By contrast, when AMPK activation was blocked, mitochondrial autophagy was decreased and cardiomyocyte apoptosis was increased. In conclusion, in the
\end{abstract}

Correspondence to: Professor Yingbin Xiao or Dr Zhao Jian, Department of Cardiovascular Surgery, Xinqiao Hospital, The Third Military Medical University, 183 Xinqiao Street, Chongqing 400037, P.R. China

E-mail:xiaoyb@tmmu.edu.cn

E-mail: zjian918@yahoo.com

*Contributed equally

Key words: adenosine monophosphate-activated protein kinase, mitophagy, mitochondrial quality control, cardioprotection, chronic hypoxia present study, mitophagy was activated and played a crucial role in cardioprotection under chronic hypoxia. AMPK was involved in mitophagy regulation, thereby providing a potential therapeutic target for heart diseases associated with chronic hypoxia.

\section{Introduction}

The heart is a highly aerobic organ $(1,2)$. Any cause of hypoxia may have a deep impact on cardiac function, and the majority of heart diseases are associated with cardiac hypoxia. Among the well-defined diseases associated with cardiac hypoxia, congenital heart disease (CHD), particularly cyanotic cardiac defects, including tetralogy of Fallot and Ebstein's anomaly (3), is one of the most common heart diseases associated with chronic and systemic hypoxemia. In addition to myocardial infarction and cardiac hypertrophy, local hypoxia promotes the progression of heart failure. Therefore, understanding the molecular changes of cardiomyocytes under chronic hypoxia may assist in the improvement of the clinical treatment of heart disease.

In cardiac tissue, chronic hypoxia triggers oxygensensitive transcriptional modulators to increase carbohydrate metabolism, enhance mitochondrial respiratory capacity and increase the efficiency of mitochondrial energy production. Therefore, the mitochondria serve a pivotal role in cardiac intrinsic regulation under hypoxia (4). Mitochondria take up at least $30 \%$ of the volume of cardiomyocytes. It has been demonstrated that peroxisome proliferator-activated receptor- $\gamma$ coactivator- $1 \alpha$ (PGC- $1 \alpha)$ is upregulated resulting in subsequent increase of mitochondrial biogenesis under chronic hypoxia (5). However, in the process of mitochondria increase, the level of reactive oxygen species (ROS) is increased by mitochondria as byproducts, which can lead to mitochondrial oxidative damage and mitochondrial dysfunction (6). Defective mitochondria enhance ROS emission, which may result in protein, nucleic acid and liquid damage, inducing oxidative stress, cellular damage and eventually cell death (7). Therefore, mitochondrial quality control is considered to be critical for the maintenance of the appropriate mitochondrial quality and quantity for cellular health. 
However, the regulatory mechanisms responsible for the mitochondrial quality control under chronic hypoxia are complex and not completely understood.

Autophagy is a catabolic process that is responsible for the degradation of cellular components via the lysosome. The process serves pivotal cellular physiological functions, including degradation of unwanted proteins, organelle turnover, response to nutrient depletion and extension of lifespan (8). The selective targeting of mitochondria by autophagy is known as mitophagy, and it eliminates unwanted or damaged mitochondria to relieve oxidative stress, thereby favoring adaptation in response to hypoxic stress (9). Accordingly, mitochondrial autophagy or mitophagy may be one of the underlying mechanisms to promote cardiomyocyte survival under hypoxic conditions.

Adenosine monophosphate-activated protein kinase (AMPK), an energy sensor and regulator, serves an important role in maintaining intracellular homoeostasis. AMPK activation can increase the uptake of glucose, enhance fatty acid oxidation (10) and inhibit protein synthesis to reserve energy stores (11). Furthermore, it has been demonstrated that hypoxia triggers AMPK activation through the ROS-mediated opening of calcium release-activated calcium channels (12). Importantly, AMPK can regulate cellular survival through autophagic regulation. A recent study reported that AMPK-mediated autophagy is essential for the maintenance of skeletal muscle function, which contains long-lived post-mitotic cells (13).

In the present study, we aim to confirm that mitophagy is critical for the quality control of mitochondria and cardiomyocyte survival, and AMPK is the key regulator of mitophagy under chronic hypoxia. Furthermore, we want to demonstrate that modulating the activity of AMPK is a therapeutic strategy for heart disease under hypoxia.

\section{Materials and methods}

Patients. A total of 20 patients with CHD, 1-28 years old (mean, 6.02 years old), were eligible for inclusion in the present study. All patients were admitted to the Institute of Cardiovascular Surgery, Xinqiao Hospital of the Third Military Medical University (Chonqing, China). Of these, 10 patients presented with a cyanotic defect and 10 with an acyanotic defect. The local Ethics Review Board of the Third Military Medical University Affiliated Hospital approved the study protocol and the informed consent forms. This investigation conforms to the principles outlined in the Declaration of Helsinki. Written informed consent was obtained from the parents of all participants.

Cardiac surgery and sampling of myocardial biopsies. Human samples were sectioned and embedded as previously described (14). Briefly, a myocardial biopsy specimen was obtained from the right ventricular outflow tracts during right ventricular outflow tract reconstruction. The myocardial samples intended for western blotting and quantitative polymerase chain reaction (qPCR) analysis were immediately snap-frozen in liquid nitrogen and stored at $-80^{\circ} \mathrm{C}$ until analysis.

Cell culture and treatment. The embryonic rat heart-derived H9c2 cells purchased from the American Type Culture Collection (CRL-1466) were grown in Dulbecco's modified Eagle's medium (Gibco; Thermo Fisher Scientific, Inc. Waltham, MA, USA) supplemented with $10 \%$ fetal bovine serum (Biological Industries, Beti-Haemek, Israel) at $37^{\circ} \mathrm{C}$ and exposed to $21 \% \mathrm{O}_{2}, 5 \% \mathrm{CO}_{2}$ and $74 \% \mathrm{~N}_{2}$. Following serum starvation overnight, cells in the hypoxic group were placed in an in vivo 200 cultivator (Ruskinn Technology, Ltd., Bridgend, $\mathrm{UK}$ ) containing a gaseous mixture of $94 \% \mathrm{~N}_{2}, 5 \% \mathrm{CO}_{2}$ and $1 \%$ $\mathrm{O}_{2}$ at $37^{\circ} \mathrm{C}$ for $48 \mathrm{~h}$. In addition, the AMPK activation group was treated with AICAR (1 mM; Sigma-Aldrich; Merck KGaA, Darmstadt, Germany), an AMPK agonist, while the inhibition group was exposed to compound $\mathrm{C}(1 \mu \mathrm{M}$; Merck KGaA), an AMPK inhibitor. Cells in the normoxic groups were incubated under the same conditions, except with $21 \% \mathrm{O}_{2}$ concentrations.

$q P C R$ analysis. Total DNA was isolated from $\mathrm{H} 9 \mathrm{c} 2$ cells and myocardial samples using DNAiso Plus (Takara Biotechnology Co., Ltd., Dalian, China) according to the manufacturer's instructions. For the quantitative determination of mitochondrial DNA (mtDNA) content relative to nuclear DNA (nDNA), primers specific for amplification of mtDNA Cyt and nDNA-encoded GAPDH were selected. The following primer sequences were used: Cyt sense, 5'-CCGGAGCAATCCAGG TCGGTT-3' and antisense, 5'-TGGTTGGGAGCACTTATG GTAAGGA-3'; and GAPDH sense, 5'-TGGGGAAGGTGA AGGTCGGAGT-3' and antisense, 5'-CCCGTTCTCAGCCTT GACGGTG-3'. SYBR ${ }^{\circledR}$ Premix Ex Taq ${ }^{\mathrm{TM}}$ II (Takara Biotechnology Co., Ltd.) was used according to the manufacturer's instructions, and qRT-PCR was performed using a StepOnePlus $^{\text {TM }}$ Real-Time PCR system (Applied Biosystems; Thermo Fisher Scientific, Inc.). The reaction conditions were as follows: $95^{\circ} \mathrm{C}$ for $30 \mathrm{sec}$, followed by 45 cycles of $95^{\circ} \mathrm{C}$ for $5 \mathrm{sec}$ and $60^{\circ} \mathrm{C}$ for $30 \mathrm{sec}$. Relative expression of target mtDNA was normalized to GAPDH using the $\Delta \Delta \mathrm{Cq}$ method (15).

Immunofluorescence. The cells were treated with or without chloroquine (Chl; $10 \mu \mathrm{M}$; Sigma-Aldrich; Merck KGaA), which inhibits fusion of autophagosomes with lysosomes (16), for $4 \mathrm{~h}$ prior to incubation at $37^{\circ} \mathrm{C}$ for $30 \mathrm{~min}$ in media containing MitoTracker (1:4,000; Life Technologies; Thermo Fisher Scientific, Inc.), then fixed with $4 \%$ formaldehyde for $15 \mathrm{~min}$ at room temperature and blocked with PBS containing $10 \%$ goat serum, 0.3 M glycine, 1\% BSA and 0.3\% Triton X-100 (Beyotime Institute of Biotechnology, Beijing, China) for $1 \mathrm{~h}$ at room temperature. Staining of the treated cells with rabbit anti-rat microtubule-associated proteins $1 \mathrm{~B}$ light chain $3 \mathrm{~B}$ (LC3B) polyclonal antibody (1:200; L7543; Sigma-Aldrich) was performed overnight at $4^{\circ} \mathrm{C}$ in PBS containing $1 \%$ BSA and $0.3 \%$ Triton X-100. Alexa Fluor 488 anti-rabbit antibody (1:500; A0423; Beyotime Institute of Biotechnology) was used as the secondary antibody incubated for $1 \mathrm{~h}$ at room temperature. A confocal laser scanning microscope (Leica TCS SP2; Leica Microsystems, Wetzlar, Germany) was used to image stained cells, and the co-localization dots were analyzed using ImageJ software (v1.6.0; National Institutes of Health, Bethesda, MD, USA). Each group contained three dependent samples, and every sample was analyzed in five random fields.

Mitochondrial potential analysis. The adherent cells were incubated with MitoTracker for $30 \mathrm{~min}$ as aforementioned, followed by treatment with rhodamine (5 $\mu \mathrm{M}$; Beyotime 
Institute of Biotechnology) for $20 \mathrm{~min}$. A confocal laser scanning microscope was used to image the stained cells. Five random fields were analyzed per sample.

JC-1 staining was also used to detect alterations of mitochondrial transmembrane potential $(\Lambda \Psi \mathrm{m})$. Cells were collected, washed and suspended in $\mathrm{JC}-1$ binding buffer (Beyotime Institute of Biotechnology). JC-1 dye (1:100; Beyotime Institute of Biotechnology) was added and the cells were incubated for $20 \mathrm{~min}$ at $37^{\circ} \mathrm{C}$ in the dark, followed by washing 2 times with binding buffer. The cell suspensions were analyzed by flow cytometry.

Apoptosis analysis. Cell apoptosis was determined by Annexin V-fluorescein 5-isothiocyanate (Annexin V-FITC) assay. H9c 2 cells ( $1 \times 10^{7}$ cells in $\left.100 \mu \mathrm{l}\right)$ were collected, washed and suspended in Annexin V binding buffer (Wanleibio Co., Ltd., Shanghai, China). FITC-conjugated Annexin V and propidium iodide (PI) (1:100; Wanleibio Co., Ltd.) were added to the binding buffer that contained suspended cells, both incubated for 15 min following the manufacturer's protocols. Following incubation, the cells were analyzed by flow cytometry (MoFloTMXDP; Beckman Coulter, Co., Fullerton, CA, USA).

Cell proliferation assay. H9c2 cells were collected from the cultures and replated into a 96 -well plate $\left(1 \times 10^{5}\right.$ cells $\left./ \mathrm{ml}\right)$. The cells in the hypoxic group, the AMPK activation group and the AMPK inhibition group were treated as described above. Cell proliferation was measured by Cell Counting Kit-8 (CCK-8; Wuhan Bioengineering Institute, Wuhan, China) assays according to the manufacturer's protocols. Prior to reading the absorbance at $450 \mathrm{~nm}, 10 \mu \mathrm{l}$ CCK-8 solution and $100 \mu \mathrm{l}$ complete medium were sequentially added to each well.

Western blotting. H9c2 cells and myocardial samples were lysed in ice-cold lysis buffer (radioimmunoprecipitation assay), with a protease inhibitor (0.5 mM PMSF) (both Beyotime Institute of Biotechnology) and phosphatase inhibitor (1 tablet/10ml; Roche Diagnostics $\mathrm{GmbH}$, Mannheim, Germany). The lysates were further centrifuged at $15,000 \mathrm{x}$ g for $15 \mathrm{~min}$ at $4^{\circ} \mathrm{C}$. Proteins from the $\mathrm{H} 9 \mathrm{c} 2$ cells and myocardial samples lysates were measured using the Bradford method. Cell proteins $(20 \mu \mathrm{g})$ were extracted with SDS lysis buffer and separated by $10 \%$ SDS-PAGE gel (both from Beyotime Institute of Biotechnology). Subsequently, protein samples were transferred to $0.22-\mu \mathrm{m}$ polyvinylidene difluoride membranes (Roche Diagnostics $\mathrm{GmbH}$ ), which were blocked with 5\% BSA for $1 \mathrm{~h}$ at room temperature, followed by incubation with primary antibodies at $4^{\circ} \mathrm{C}$ overnight. The primary antibodies included the following: Rabbit anti-mouse AMPK $\alpha$ polyclonal antibody (1:1,000; \#5831), rabbit ant-mouse p-AMPK $\alpha$ polyclonal antibody (1:1,000; \#2535) (both Sigma-Aldrich; Merck KGaA) and rabbit anti-mouse $\beta$-actin monoclonal antibody $(1: 1,000$; bs-0061R; Bioss, Beijing, China). Next, the membranes were incubated with horseradish peroxidase-conjugated secondary antibodies (1:5,000; A0208) for $1 \mathrm{~h}$ at $37^{\circ} \mathrm{C}$ and detected using an enhanced chemiluminescence kit (both from Beyotime Institute of Biotechnology), and densitometry signals were quantified using ImageQuant TL software (General Electric, Co., Fairfield, CT, USA).
Table I. Clinical characteristics of included patients.

\begin{tabular}{|c|c|c|}
\hline Characteristic & Acyanotic & Cyanotic \\
\hline $\mathrm{n}$ & 10 & 10 \\
\hline Age at surgery, years ${ }^{\mathrm{a}}$ & $6.17 \pm 5.98$ & $5.86 \pm 8.64$ \\
\hline Gender (male/female), $\mathrm{n}^{\mathrm{a}}$ & $4 / 6$ & $3 / 7$ \\
\hline $\mathrm{SaO}_{2}, \% \mathrm{a}$ & $97.30 \pm 1.89$ & $71.30 \pm 11.83^{\mathrm{b}}$ \\
\hline $\mathrm{Hb}, \mathrm{g} / \mathrm{dl}^{\mathrm{a}}$ & $12.31 \pm 0.55$ & $16.41 \pm 3.32^{b}$ \\
\hline Hct, $\%$ a & $37.65 \pm 1.64$ & $51.24 \pm 9.48^{b}$ \\
\hline \multicolumn{3}{|l|}{ Pathology, n } \\
\hline TOF & & 8 \\
\hline VSD and PA & & 2 \\
\hline VSD and RVOS & 10 & \\
\hline
\end{tabular}

${ }^{\mathrm{a} D a t a}$ are presented as mean $\pm \mathrm{SD}$. ${ }^{\mathrm{b}} \mathrm{P}<0.01$ vs. acyanotic group. $\mathrm{SaO}_{2}$, transcutaneous arterial oxygen saturation; Hb, hemoglobin; Hct, hematocrit; Acyanotic group, acyanotic congenital heart diseases; Cyanotic group, cyanotic congenital heart diseases; TOF, tetralogy of Fallot; VSD, ventricular septum defect; PA, pulmonary atresia; RVOS, right ventricular outflow tract obstruction.

Statistical analysis. Data from at least three independent experiments are expressed as the mean \pm SD. SPSS 16.0 software (SPSS, Inc., Chicago, IL, USA) was used for the statistical analysis. The difference in means between 2 groups was evaluated using Student's t-test, and one-way analysis of variance was used for comparisons between $\geq 2$ groups. $\mathrm{P}<0.05$ was considered to indicate a statistically significant difference.

\section{Results}

Hypoxia increases mitochondrial biogenesis, $\Lambda \Psi m$ loss and cell apoptosis in $H 9 c 2$ cells. The epidemiological data of the patients is summarized in Table I. Patients with cyanotic heart disease had significantly lower preoperative arterial oxygen saturation $\left(\mathrm{SaO}_{2}\right)$ than patients with acyanotic heart disease. Comparing the cyanotic patients with the acyanotic patients, the relative mtDNA/nDNA ratio was found to be higher in the cyanotic group. Similarly, the ratio of $\mathrm{H} 9 \mathrm{c} 2$ cells in the hypoxic group was higher than that in the H9c2 cells cultured under normoxic conditions. The copies of mtDNA Cyt were significantly increased in the cyanotic patients and the H9c2 cells of hypoxic group when compared with the acyanotic patients and the H9c2 cells of the normoxic group, respectively (Fig. 1A). Fluorescence microscopy was performed to detect $\Lambda \Psi \mathrm{m}$ in the cells cultivated under hypoxic and normoxic conditions. Cells were co-stained with rhodamine 123 and MitoTracker, and it was found that the transmembrane potential was decreased in hypoxic cardiomyocytes, as the co-localization of rhodamine 123 and MitoTracker was decreased (Fig. 1B). Moreover, after the cells were stained with JC-I, which more specifically reduces mitochondrial $\Lambda \Psi \mathrm{m}$, the loss of $\Lambda \Psi \mathrm{m}$ was increased and the number of $\mathrm{H} 9 \mathrm{c} 2$ cells with intact $\Lambda \Psi \mathrm{m}$ was reduced compared with the normoxic H9c2 cells (Fig. 1C). Loss of the $\Lambda \Psi \mathrm{m}$ is a key step in the intrinsic apoptotic pathway (17). Therefore, the apoptosis of H9c2 cells was 
A

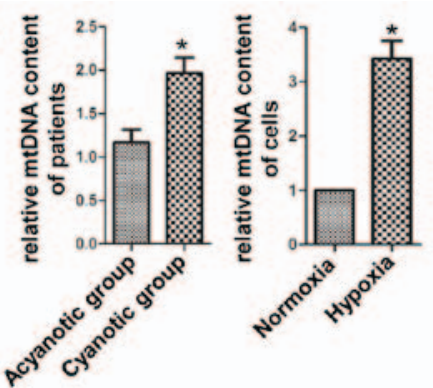

B

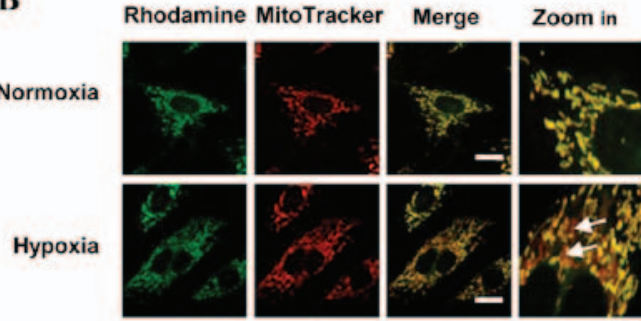

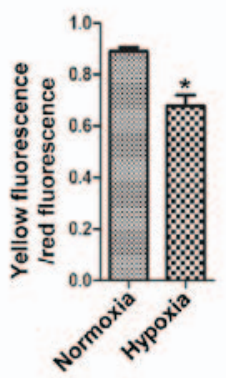

C

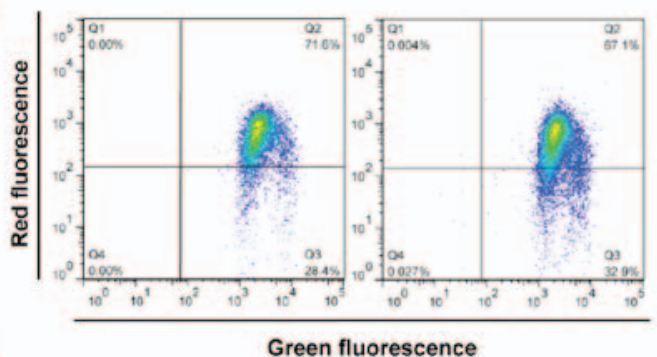

D

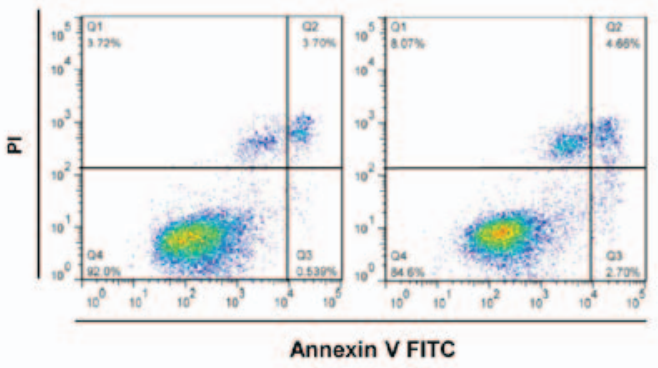

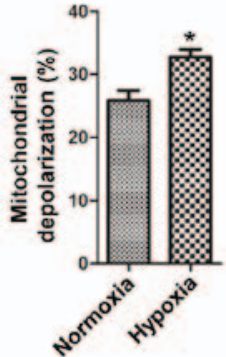

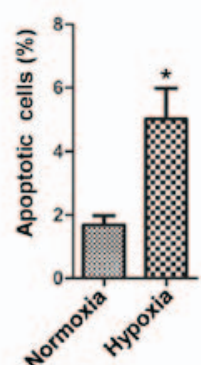

E

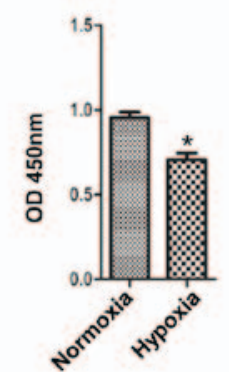

Figure 1. Mitochondrial biogenesis is increased under hypoxic conditions, with mitochondria membrane potential ( $\Lambda \Psi \mathrm{m})$ loss and cell apoptosis. H9c2 cells were cultured in 1 or $21 \% \mathrm{O}_{2}$ and $5 \% \mathrm{CO}_{2}$ for $48 \mathrm{~h}$. (A) Quantitative polymerase chain reaction revealed that the mtDNA copy number was significantly increased in the myocardium of congenital heart disease patients and $\mathrm{H} 9 \mathrm{c} 2$ cells under hypoxic conditions ( $\mathrm{P}<0.05$ vs. acyanotic or normoxic group). (B) $\Lambda \Psi \mathrm{m}$ was markedly deceased in the hypoxic state, which was assayed using laser confocal microscopy following mitochondrial tracker (red) and rhodamine (green) immunofluorescence double-labeled staining (arrows indicate the mitochondrial with reduced $\Lambda \Psi \mathrm{m}$; scale bar, $20 \mu \mathrm{m}$ ). Quantified by yellow fluorescence intensities as percentage relative to red florescence intensities ( $n=3 ;{ }^{*} \mathrm{P}<0.05$ vs. normoxic group). (C) The percentage of $\mathrm{H} 9 \mathrm{c} 2$ cells with reduced $\Lambda \Psi \mathrm{m}$ was evaluated using flow cytometry following JC-1 staining. The proportion of cells with decreased $\Lambda \Psi \mathrm{m}$ in the hypoxic group was higher than that in the normoxic group ( $\mathrm{n}=3$; ${ }^{*} \mathrm{P}<0.05 \mathrm{vs}$. normoxic group). (D) Cell apoptosis was measured with Annexin V and PI staining by flow cytometry, and the proportion of Annexin V-positive cells in the hypoxic group was higher than that in the normoxic group ( $\mathrm{n}=3 ;{ }^{*} \mathrm{P}<0.05$ vs. normoxic group). (E) Cell Counting Kit- 8 assay was used to measure the proliferation of the H9c2 cells, the OD value exhibited a significant decrease in the hypoxic group compared with that in the normoxic group ( $\mathrm{n}=3$; ${ }^{*} \mathrm{P}<0.05 \mathrm{vs}$. normoxic group). Data are expressed as the mean \pm standard error of the mean of 3 independent experiments. PI, propidium iodide; FITC, fluorescein isothiocyanate; OD, optical density.
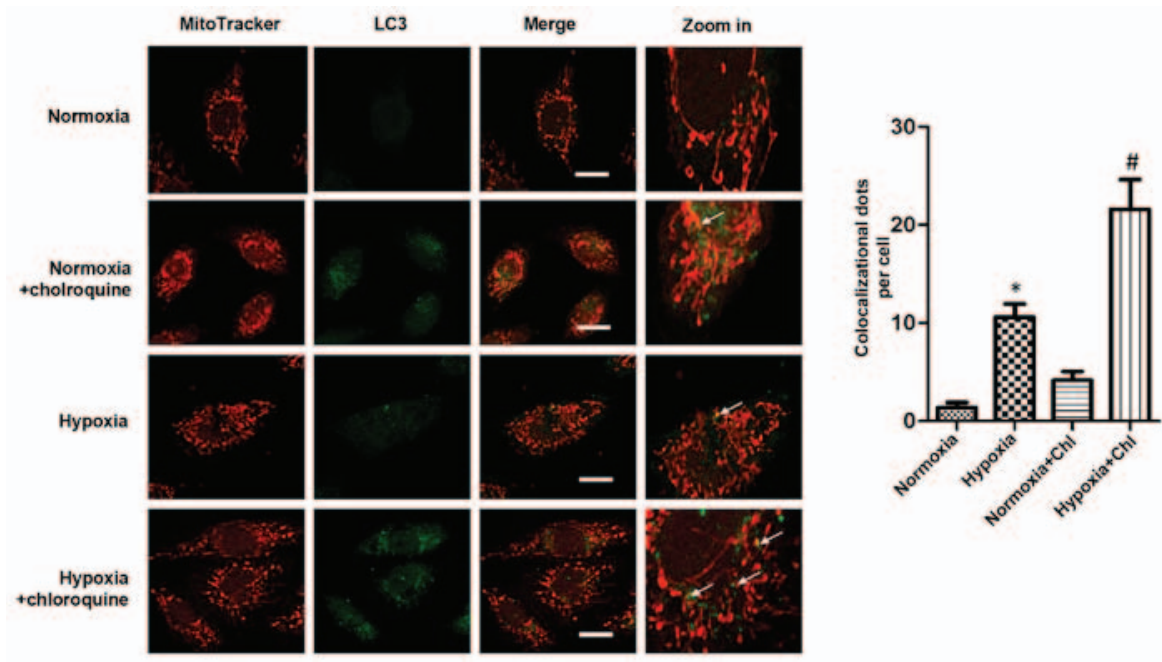

Figure 2. Mitochondrial autophagy is facilitated under hypoxia in H9c2 cells. Representative imaging of mitochondria tracker (red) and LC3 dots (green) confocal images of normoxic and hypoxic group, with or without Chl $(10 \mu \mathrm{M})$. Arrows indicate LC3 puncta that colocalize with mitochondria (scale bar, $20 \mu \mathrm{m})$. The number of dots of green and red colocalization were significantly increased in the hypoxic group compared with that in the normoxic group. (C) Quantification of the mitophagy based on the LC3 dots (green) colocalized with mitochondria (red). ( $\mathrm{P}<0.05$ vs. normoxic group; ${ }^{*} \mathrm{P}<0.05$ vs. normoxic group treated with Chl). Data are expressed as the mean \pm standard error of the mean of 3 independent experiments. LC3, light chain 3; Chl, chloroquine. 

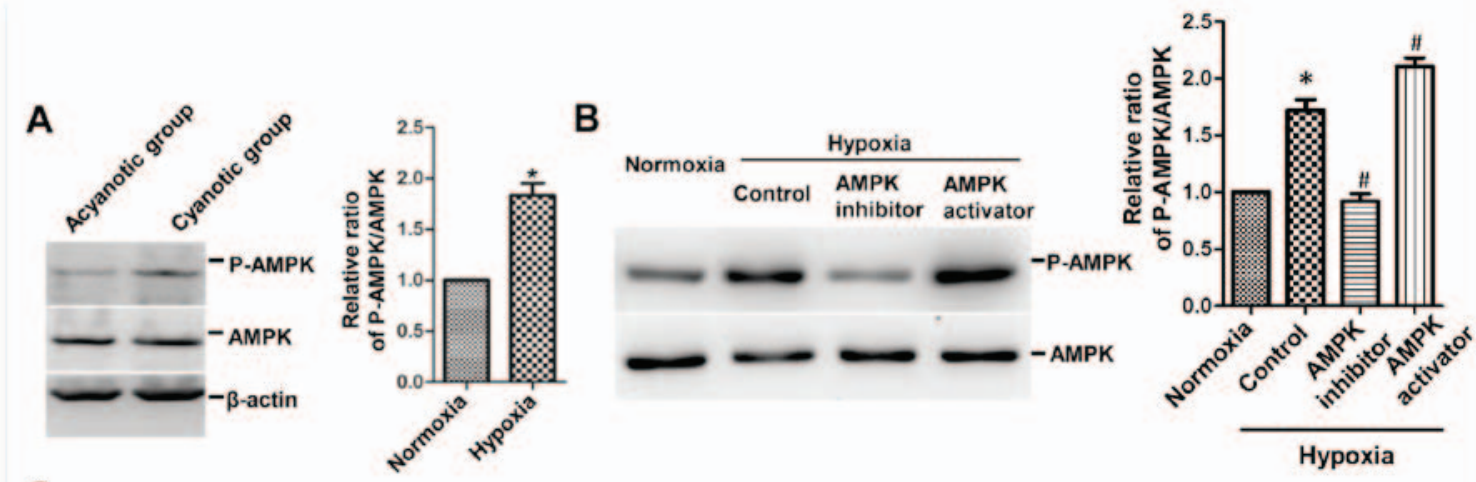

C

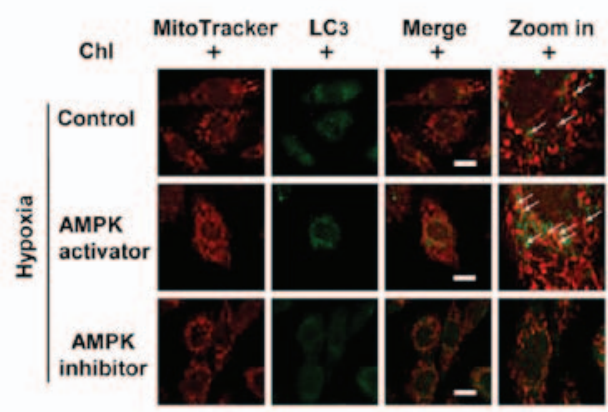

D
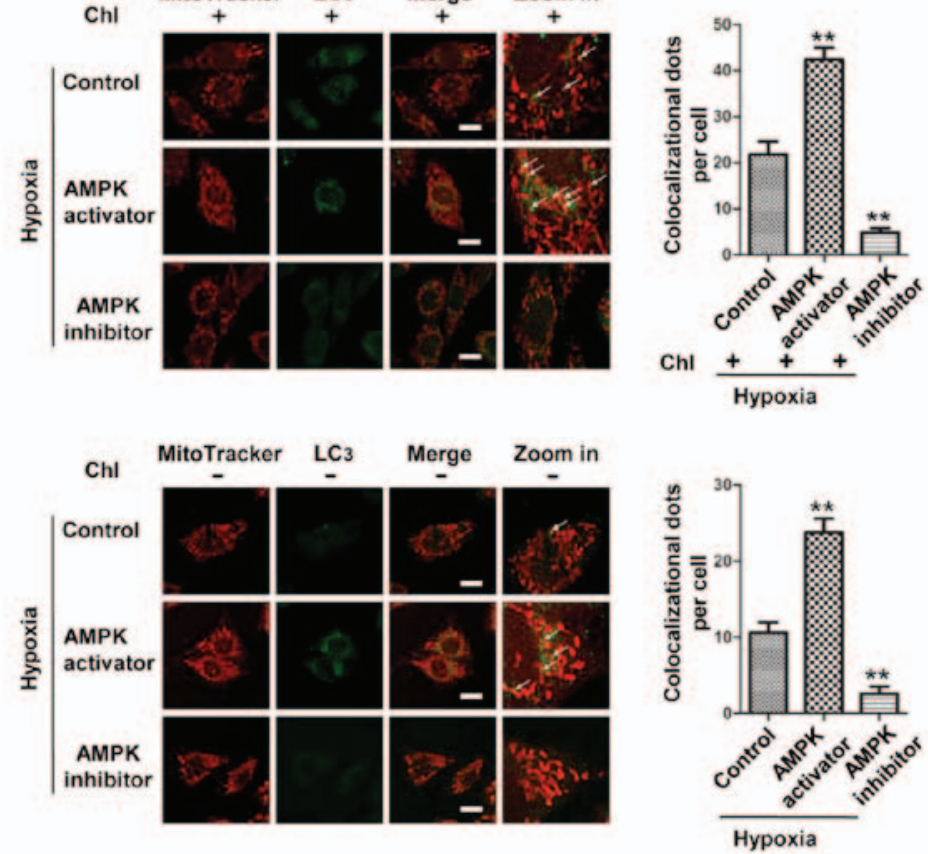

Figure 3. Activation of AMPK is critical for mitophagy under hypoxia. (A) Immunoblot analysis indicated that the ratio of phosphorylated AMPK expression to AMPK expression in the myocardium of congenital heart disease patients was higher than that in patients with acyanotic heart disease $($ P $<0.05$ vs. normoxic group). (B) Representative images of western blotting and summarized data showing that the ratio of phosphorylated AMPK expression to AMPK expression was higher in the hypoxic group compared with that in the normoxic group, and that the ratio was higher in the AMPK activation group, which was treated with AICAR ( $1 \mathrm{mM})\left({ }^{*} \mathrm{P}<0.05\right.$ vs. normoxic group), and lower in the AMPK inhibition group, which was treated with compound $\mathrm{C}(10 \mu \mathrm{M})\left({ }^{\#} \mathrm{P}<0.05\right.$ vs. hypoxic group). (C and D) Representative images of mitochondria tracker (red) and LC3 dots (green) in cells treated with or without Chl (10 $\mu \mathrm{M})$. Arrows indicate LC3 puncta colocalized with mitochondria (scale bar, $20 \mu \mathrm{m}$ ). Quantification of the mitophagy level based on the LC3 dots (green) colocalized with mitochondria (red). The number of dots of green and red colocalization were significantly increased in the AMPK activation group and decreased in the AMPK inhibition group. ( $\mathrm{n}=3$; ${ }^{* *} \mathrm{P}<0.01$ vs. control). p-AMPK, phosphorylated adenosine monophosphate-activated protein kinase; LC3, light chain 3; Chl, chloroquine.

evaluated using flow cytometry following staining of the cells with Annexin V-FITC in combination with PI. A significantly higher percentage of apoptotic cells were detected in the hypoxic group (Fig. 1D). The CCK-8 assay showed that the proliferation of the H9c2 cells was significantly decreased in the hypoxic group compared with that in the normoxic group (Fig. 1E). The results revealed that compared with that in acyanotic patients, mitochondrial biogenesis was upregulated in patients with cyanotic heart disease and in the $\mathrm{H} 9 \mathrm{c} 2$ cells cultivated in the chronic hypoxic condition. In addition to the upregulated mitochondrial biogenesis, the loss of $\Lambda \Psi \mathrm{m}$ and the apoptosis of the cells were also significantly increased compared with the normoxic cells. These data suggested that chronic hypoxia promoted mitochondrial biogenesis and induced mitochondrial damage and cell apoptosis at the same time.
Hypoxia facilitates mitophagy. As the damaged and dysfunctional mitochondria increased in the $\mathrm{H} 9 \mathrm{c} 2$ cells exposed to long periods of hypoxia, selective mitochondrial autophagy was detected in cells exposed to hypoxia. After staining the cells with antibody to LC3B and immunofluorescence assessment, it was found that LC3B rarely localized in the mitochondria, confirming the presence of selective mitochondrial autophagy, also known as mitophagy, in the cardiomyocytes. Notably, the expression of LC3 was increased in the mitochondria when the $\mathrm{H} 9 \mathrm{c} 2$ cells were subjected to long periods of hypoxia compared with that in cardiomyocytes cultured under normoxic conditions. Moreover, when the cardiomyocytes were treated with Chl, an inhibitor of autophagosome-lysosome fusion, the expression of LC3 in the mitochondria was significantly increased (Fig. 2). These results suggested that mitophagosomes were increased in the 
A

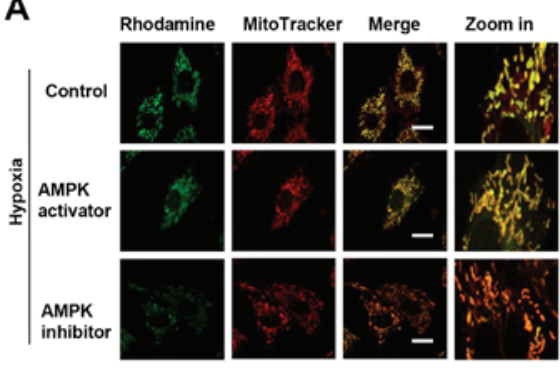

B

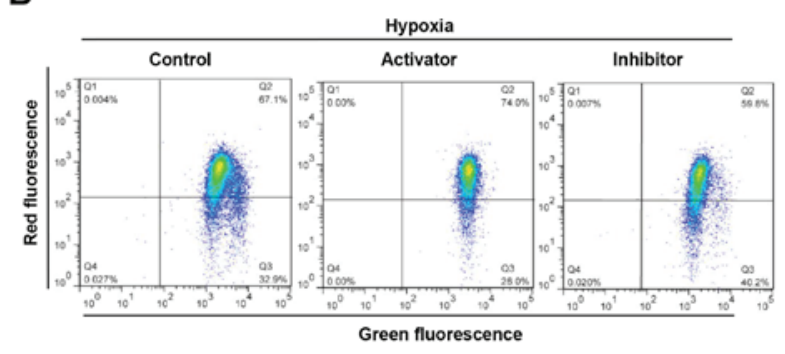

C

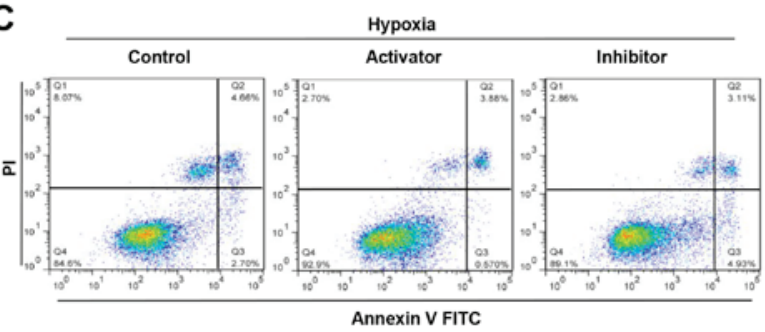

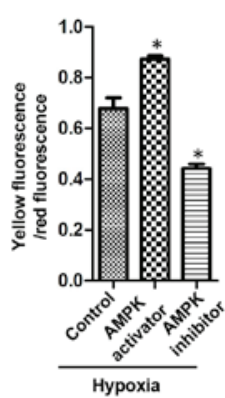
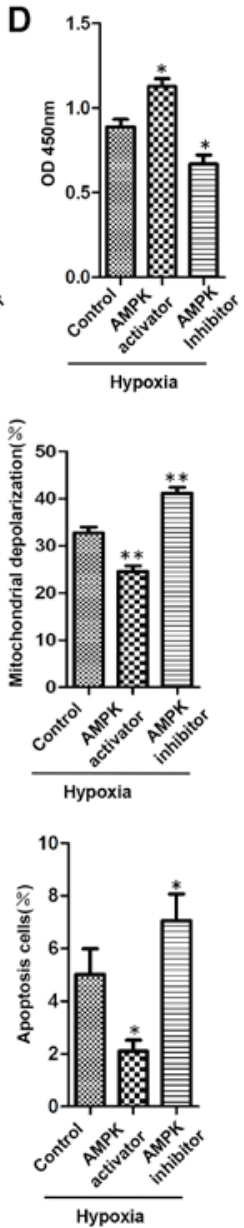

Figure 4. AMPK activation contributes to mitochondrial quality control and promotes H9c2 cell survival and proliferation under hypoxia. (A) Rhodamine fluorescence (green) and MitoTracker (red) analysis was used to detected the mitochondrial membrane potential ( $\Lambda \Psi \mathrm{m})$, the results indicated that the loss of $\Lambda \Psi \mathrm{m}$ was increased in the AMPK activation group and decreased in the AMPK inhibition group ( $\mathrm{n}=3 ;{ }^{* *} \mathrm{P}<0.05$ vs. control). (B) Percentages of $\Lambda \Psi \mathrm{m}$ decreased cells were detected by flow cytometry using JC-1 dye, and the relative proportion of $\Lambda \Psi \mathrm{m}$ decreased cells in the AMPK activation group was higher than that in the AMPK inhibition group ( $\mathrm{n}=3 ;{ }^{* *} \mathrm{P}<0.01 \mathrm{vs}$. control). (C) Cell apoptosis was assayed using flow cytometry following Annexin $\mathrm{V}$ and PI staining demonstrated that cell apoptosis was decreased in the AMPK activator group, but increased in the AMPK inhibitor group ( $\mathrm{n}=3 ;{ }^{*} \mathrm{P}<0.05$ vs. control). (D) Cell viability of each group measured by Cell Counting Kit-8 indicated that AMPK activation significantly increases cell viability, while inhibition of AMPK shows the opposite results ( $\mathrm{n}=3$; ${ }^{*} \mathrm{P}<0.05$ vs. control). AMPK, adenosine monophosphate-activated protein kinase; PI, propidium iodide; FITC, fluorescein isothiocyanate.

H9c2 cells under hypoxic conditions and that the mitophagy was induced by chronic hypoxia.

Hypoxia induces the activation of AMPK, and inhibited AMPK activation decreases mitophagy in cardiomyocytes. Since AMPK plays a significant role in maintaining intracellular homoeostasis during hypoxia, the present study first investigated the impact of chronic hypoxia on AMPK activation. As expected, western blotting results confirmed that the level of phosphorylation of AMPK was increased in the ventricular myocardium of cyanotic patients and in the H9c2 cells cultured under hypoxic conditions (Fig. 3A and B). To test further whether AMPK was necessary in regulating mitophagy, the activation of AMPK was determined following different treatments, including AMPK activator AICAR and AMPK inhibitor compound $\mathrm{C}$, and it was found that the level of phosphorylation of AMPK was increased in the AMPK activation group and decreased in the AMPK inhibition group compared with that in the hypoxic control group (Fig. 3B). Immunofluorescence showed that the AMPK activator increased the co-localization of LC3 dots (green) and mitochondria (red) in the Chl-treated and untreated H9c2 cells, while the AMPK inhibitor presented the opposite results (Fig. 3C and D). These results demonstrated that the activity of AMPK was increased in the myocardium of infants with cyanotic cardiac defects and in the H9c2 cells under hypoxia. In addition, mitophagy was promoted in the cells of the AMPK activation group and suppressed in the AMPK inhibition group, thereby suggesting that activation of AMPK is a critical factor for mitophagy under chronic hypoxia.

AMPK activation contributes to mitochondrial quality control and promotes $\mathrm{H} 9 \mathrm{c} 2$ cell survival. To determine whether AMPK contributes to mitochondrial quality control and protects cardiomyocytes during chronic hypoxia, $\Lambda \Psi \mathrm{m}$ was detected using fluorescence microscopy following rhodamine 123 and MitoTracker staining. Compared with that of the hypoxic control group, the percentage of dysfunctional mitochondria was decreased in the AMPK activation group, but increased in the AMPK inhibition group (Fig. 4A). Following the JC-1 staining, flow cytometry revealed that the number of $\mathrm{H} 9 \mathrm{c} 2$ cells with reduced $\Lambda \Psi \mathrm{m}$ was decreased in the AMPK activation 
group and increased in the AMPK inhibition group compared with that of the hypoxic group (Fig. 4B). The apoptosis of H9c2 cells was detected using flow cytometry following staining with Annexin V-FITC in combination with PI. It was observed that, compared with that in the control group, the ratio of early apoptotic cells was decreased following treatment of AICAR, but increased following treatment with compound C (Fig. 4C). The proliferation of $\mathrm{H} 9 \mathrm{c} 2$ cells that was detected by CCK-8 assay was increased by AMPK activator, but decreased by AMPK inhibitor (Fig. 4D). These data suggested that under chronic hypoxic conditions, activation of AMPK contributed to the mitochondrial quality and has a protective role for cell survival.

\section{Discussion}

In the present study, mitophagy was found to be upregulated under hypoxic conditions, with AMPK activity being increased accordingly. AMPK played a critical role in the regulation of mitophagy under hypoxic conditions. Mitochondrial function is key for maintaining cardiomyocyte function, and extensive mitochondrial damage can induce apoptosis via different pathways $(18,19)$. Mitochondria are dynamic organelles that have unique features, including their own genome and a maternal mode of germ line transmission (20). To ensure the integrity of mitochondria and their DNA, mitochondria undergo fusion-fission dynamics, organelle transport, genetic selection of functional genomes and mitochondrial autophagy or mitophagy (21). Mitochondrial fusion and fission allows exchange of mitochondrial components and segregation of terminally damaged mitochondria, to ensure more effective degradation of unwanted and damaged mitochondria by specific mitophagy (22). Mitophagy not only recycles the components of the mitochondria, but also selectively eliminates the damaged mitochondria to regulate their quantity and quality (23). This significantly contributes to cellular mitochondrial quality control and maintaining mitochondrial function, and even cell survival.

In recent years, intensive studies on the regulation and function of AMPK have indicated that AMPK acts as a 'fuel gauge' in numerous types of cells. AMPK is important in maintaining intracellular homoeostasis during many stressful challenges, including hypoxia, and regulates the ROS/redox balance, cell proliferation, cell apoptosis, autophagy, mitochondrial function and genotoxic response (24). It has been well established that AMPK can regulate ROS through the regulation of autophagy (25). In the heart, AMPK restores the adenosine triphosphate supply for cardiomyocytes and plays an important role in physiological states and stress conditions. A number of studies have indicated that AMPK activation can protect cardiomyocytes and limit the damage from myocardial ischemic injury $(26,27)$.

It has been demonstrated that hypoxia leads to mitochondrial damage and dysfunction, including decreased oxidative phosphorylation, released apoptogenic factors, such as cytochrome $c$ oxidase, and increased ROS regeneration $(7,28)$. Hypoxia also triggers AMPK activation to deal with stress challenge. The present study observations indicate that increased AMPK activation could play a protective role in allowing cardiomyocytes to survive under chronic hypoxic stress by stimulating mitophagy, which ensures mitochondrial quality control.
Under hypoxic conditions, hypoxia-inducible factor-1 is induced and mediates the induction of B-cell lymphoma 2 (Bcl-2)/adenovirus E1B 19 kDa-interacting protein 3 (BNIP3), which is usually described as a pro-apoptotic member of the Bcl-2 family that triggers selective mitochondrial autophagy (29). Moreover, it has been demonstrated that several mitophagy receptors, including BNIP3, NIX (also known as BNIP3L) and mitochondrial outer membrane protein FUN14 domain containing 1 cooperate with the PTEN-induced putative kinase 1-Parkin pathway and play a significant role to fine-tune the mitophagy process (9). A recent study indicated that endogenous dynamin-related protein 1 serves an important role in mediating mitochondrial autophagy in cardiomyocytes (30). It is well known that a molecular mechanism for the regulation of mammalian autophagy involves UNK-51-like kinase 1 (ULK1), the mammalian homolog of yeast protein kinase Atg1. ULK1 induces autophagy by phosphorylating beclin 1 and activating Vps34 lipid kinase (31). AMPK stimulates autophagy or mitophagy by directly activating ULK1 through phosphorylation of Ser317 and Ser777, and suppressing the mammalian target of rapamycin complex 1 , which inhibits autophagy by preventing ULK1 activation by phosphorylation Ulk1 Ser757 $(32,33)$. In addition, another recent investigation indicated that the AMPK-dependent phosphorylation of ULK1 regulates the translocation of ULK1 to the mitochondria to serve a critical role in the mitophagy response to hypoxic stress (34).

In conclusion, chronic hypoxia induces the activation of AMPK, which promotes selective mitophagy to maintain mitochondrial quality and quantity for the cell. The present study demonstrated that mitophagy induced by AMPK activation may play a significant role in cardiac protection while cardiomyocytes undergo chronic hypoxia. This study provides a possible mechanism for metabolic adaptation and cardioprotection in response to chronic hypoxia; therefore, it is worthy of clinical exploration as a therapeutic strategy for heart disease caused by chronic hypoxia, including cyanotic heart disease, high-altitude heart disease and chronic cor pulmonale. The mechanism underlying AMPK-mediated mitophagy requires future investigation.

\section{Acknowledgements}

This study was supported by the National Natural and Science Foundation of China (grant no. 81270228).

\section{References}

1. Schaper J, Meiser E and Stammler G: Ultrastructural morphometric analysis of myocardium from dogs, rats, hamsters, mice, and from human hearts. Circ Res 56: 377-391, 1985.

2. Vásquez-Trincado C, García-Carvajal I, Pennanen C, Parra V, Hill JA, Rothermel BA and Lavandero S: Mitochondrial dynamics, mitophagy and cardiovascular disease. J Physiol 594: 509-525, 2016.

3. Driscoll DJ: Evaluation of the cyanotic newborn. Pediatr Clin North Am 37: 1-23, 1990.

4. Kornosky JL and Salihu HM: Getting to the heart of the matter: epidemiology of cyanotic heart defects. Pediatr Cardiol 29: 484-497, 2008

5. Zhu L, Wang Q, Zhang L, Fang Z, Zhao F, Lv Z, Gu Z, Zhang J, Wang J, Zen K, et al: Hypoxia induces PGC-1 $\alpha$ expression and mitochondrial biogenesis in the myocardium of TOF patients. Cell Res 20: 676-687, 2010 
6. Fischer F, Hamann A and Osiewacz HD: Mitochondrial quality control: an integrated network of pathways. Trends Biochem Sci 37: 284-292, 2012.

7. Held NM and Houtkooper RH: Mitochondrial quality control pathways as determinants of metabolic health. BioEssays 37: 867-876, 2015

8. Mizushima N, Levine B, Cuervo AM and Klionsky DJ: Autophagy fights disease through cellular self-digestion. Nature 451 : 1069-1075, 2008.

9. Wu $\mathrm{H}$ and Chen Q: Hypoxia activation of mitophagy and its role in disease pathogenesis. Antioxid Redox Signal 22: 1032-1046, 2015.

10. Hardie DG and Sakamoto K: AMPK: a key sensor of fuel and energy status in skeletal muscle. Physiology (Bethesda) 21: 48-60, 2006.

11. Liu L, Cash TP, Jones RG, Keith B, Thompson CB and Simon MC: Hypoxia-induced energy stress regulates mRNA translation and cell growth. Mol Cell 21: 521-531, 2006.

12. Mungai PT, Waypa GB, Jairaman A, Prakriya M, Dokic D, Ball MK and Schumacker PT: Hypoxia triggers AMPK activation through reactive oxygen species-mediated activation of calcium release-activated calcium channels. Mol Cell Biol 31: 3531-3545, 2011

13. Bujak AL, Crane JD, Lally JS, Ford RJ, Kang SJ, Rebalka IA, Green AE, Kemp BE, Hawke TJ, Schertzer JD, et al: AMPK activation of muscle autophagy prevents fasting-induced hypoglycemia and myopathy during aging. Cell Metab 21: 883-890, 2015.

14. Jian Z, Li JB, Ma RY, Chen L, Zhong QJ, Wang XF, Wang W, Hong $\mathrm{Y}$ and Xiao $\mathrm{YB}$ : Increase of macrophage migration inhibitory factor (MIF) expression in cardiomyocytes during chronic hypoxia. Clin Chim Acta 405: 132-138, 2009.

15. Livak KJ and Schmittgen TD: Analysis of relative gene expression data using real-time quantitative PCR and the 2(-Delta Delta C(T)) Method. Methods 25: 402-408, 2001

16. Iwai-Kanai E, Yuan H, Huang C, Sayen MR, Perry-Garza CN, Kim L and Gottlieb RA: A method to measure cardiac autophagic flux in vivo. Autophagy 4: 322-329, 2008.

17. Webster KA: Mitochondrial membrane permeabilization and cell death during myocardial infarction: roles of calcium and reactive oxygen species. Future Cardiol 8: 863-884, 2012.

18. Gustafsson AB and Gottlieb RA: Heart mitochondria: gates of life and death. Cardiovasc Res 77: 334-343, 2008.

19. Chiong M, Wang ZV, Pedrozo Z, Cao DJ, Troncoso R, Ibacache M, Criollo A, Nemchenko A, Hill JA and Lavandero S: Cardiomyocyte death: mechanisms and translational implications. Cell Death Dis 2: e244, 2011.

20. Chen H and Chan DC: Mitochondrial dynamics - fusion, fission, movement, and mitophagy - in neurodegenerative diseases. Hum Mol Genet 18: R169-R176, 2009.
21. Mishra P and Chan DC: Mitochondrial dynamics and inheritance during cell division, development and disease. Nat Rev Mol Cell Biol 15: 634-646, 2014.

22. Youle RJ and van der Bliek AM: Mitochondrial fission, fusion, and stress. Science 337: 1062-1065, 2012.

23. Youle RJ and Narendra DP: Mechanisms of mitophagy. Nat Rev Mol Cell Biol 12: 9-14, 2011.

24. Wang S, Song P and Zou MH: AMP-activated protein kinase, stress responses and cardiovascular diseases. Clin Sci (Lond) 122: 555-573, 2012.

25. Roach PJ: AMPK -> ULK1 -> autophagy. Mol Cell Biol 31: 3082-3084, 2011.

26. Young LH: AMP-activated protein kinase conducts the ischemic stress response orchestra. Circulation 117: 832-840, 2008.

27. Russell RR 3rd, Li J, Coven DL, Pypaert M, Zechner C, Palmeri M, Giordano FJ, Mu J, Birnbaum MJ and Young LH: AMP-activated protein kinase mediates ischemic glucose uptake and prevents postischemic cardiac dysfunction, apoptosis, and injury. J Clin Invest 114: 495-503, 2004.

28. Turrens JF: Mitochondrial formation of reactive oxygen species. J Physiol 552: 335-344, 2003.

29. Semenza GL: Mitochondrial autophagy: life and breath of the cell. Autophagy 4: 534-536, 2008.

30. Ikeda Y, Shirakabe A, Maejima Y, Zhai P, Sciarretta S, Toli J, Nomura M, Mihara K, Egashira K, Ohishi M, et al: Endogenous Drp1 mediates mitochondrial autophagy and protects the heart against energy stress. Circ Res 116: 264-278, 2015.

31. Russell RC, Tian Y, Yuan H, Park HW, Chang YY, Kim J, Kim H, Neufeld TP, Dillin A and Guan KL: ULK1 induces autophagy by phosphorylating Beclin-1 and activating VPS34 lipid kinase. Nat Cell Biol 15: 741-750, 2013.

32. Kim J, Kundu M, Viollet B and Guan KL: AMPK and mTOR regulate autophagy through direct phosphorylation of Ulk1. Nat Cell Biol 13: 132-141, 2011.

33. Egan DF, Shackelford DB, Mihaylova MM, Gelino S, Kohnz RA, Mair W, Vasquez DS, Joshi A, Gwinn DM, Taylor R, et al: Phosphorylation of ULK1 (hATG1) by AMP-activated protein kinase connects energy sensing to mitophagy. Science 331: 456-461, 2011.

34. Tian W, Li W, Chen Y, Yan Z, Huang X, Zhuang H, Zhong W, Chen Y, Wu W, Lin C, et al: Phosphorylation of ULK1 by AMPK regulates translocation of ULK1 to mitochondria and mitophagy. FEBS Lett 589: 1847-1854, 2015.

(i) $($ This work is licensed under a Creative Commons Attribution-NonCommercial-NoDerivatives 4.0 International (CC BY-NC-ND 4.0) License. 